\title{
Follow-up duration influences the relative importance of OGTT and optimal timing of glucose measurements for predicting future type 2 diabetes
}

\author{
Mette Lundgren Nielsen', Manan Pareek1, Margrét Leósdóttir², Kurt Højlund ${ }^{3,4}$, \\ Karl-Fredrik Eriksson ${ }^{5}$, Peter M Nilsson ${ }^{6}$ and Michael Hecht Olsen ${ }^{1,7}$ \\ ${ }^{1}$ Cardiovascular and Metabolic Preventive Clinic, Department of Endocrinology, Centre for \\ Individualized Medicine in Arterial Diseases (CIMA), Odense University Hospital, Odense, Denmark, \\ ${ }^{2}$ Department of Cardiology, Skåne University Hospital, Malmö, Sweden, ${ }^{3}$ Department of Endocrinology, \\ Odense University Hospital, Odense, Denmark, ${ }^{4}$ Section of Molecular Diabetes \& Metabolism, Institute \\ of Molecular Medicine \& Institute of Clinical Research, University of Southern Denmark, Odense, \\ Denmark, ${ }^{5}$ Department of Clinical Sciences, Vascular Diseases, Lund University, Malmö, Sweden, \\ ${ }^{6}$ Department of Clinical Sciences, Lund University, Skåne University Hospital, Malmö, Sweden, and \\ ${ }^{7}$ Hypertension in Africa Research Team (HART), North-West University, Potchefstroom, South Africa
}

\author{
Correspondence \\ should be addressed \\ to Mette Lundgren Nielsen \\ Email \\ mettelundgren@gmail.com
}

\begin{abstract}
Objective: To examine the impact of follow-up duration on the incremental prognostic yield of a baseline oral glucose tolerance test (OGTT) for predicting type 2 diabetes and to assess the discrimination ability of blood glucose (BG) obtained at different time points during OGTT.

Design: A prospective, population-based cohort study (Malmö Preventive Project) with inclusion of subjects from 1974 to 1992. Methods: A total of 5256 men without diabetes, who had BG measured at 0, 20, 40, 60, 90, and 120 min during OGTT ( $30 \mathrm{~g} / \mathrm{m}^{2}$ glucose), were followed for 30 years. Incident type 2 diabetes was recorded using registries. The performance of OGTT added to a clinical prediction model (age, body mass index (BMI), diastolic blood pressure, fasting BG, triglycerides, and family history of diabetes) was assessed using Harrell's concordance index (C-index) and integrated discrimination improvement (IDI). Results: Median age was 48 years, mean BMI $24.9 \mathrm{~kg} / \mathrm{m}^{2}$, and mean fasting BG $4.7 \mathrm{mmol} / \mathrm{L}$. Models with added post-load BG performed better than the clinical model (C-index: $P=0.08$ for BG at 120 min at 5 years, otherwise $P \leq 0.045$; IDI: $P \geq 0.06$ for BG at 60 and 90 min at 5 years, otherwise $P \leq 0.01$ ). With a longer follow-up duration, $C$-index decreased, and the C-index increase associated with OGTT was attenuated. Models including BG at 60 or 90 min performed significantly better than the model with BG at 120 min, evident beyond follow-up of 10 and 5 years, respectively.

Conclusions: OGTT provided incremental prognostic yield for type 2 diabetes prediction. BG measured at 60 or 90 min provided better discrimination than BG at $120 \mathrm{~min}$.

\section{Introduction}

The prevalence of type 2 diabetes is rapidly increasing and is likely to approach 600 million worldwide by 2035 (http://www.idf.org/diabetesatlas (cited 2015 July 20)). The rise is closely linked to the obesity epidemic (1) and constitutes a heavy economic burden on every health care system.
두 2016 European Society of Endocrinology Printed in Great Britain
The importance of predicting subjects at high risk for developing type 2 diabetes is further emphasized by the fact that lifestyle modification and pharmacological intervention can prevent progression into overt diabetes, at least when high risk is defined as overweight subjects with impaired glucose tolerance (IGT) detected by oral glucose

Published by Bioscientifica Ltd. 
tolerance test (OGTT) $(2,3,4)$. Nonetheless, the cost and time consumption of OGTT has prompted the development of prediction models based on easily measureable risk factors, e.g. age, gender, fasting plasma glucose (FPG), serum lipids, BP, family history of diabetes, and anthropometric measurements (5). Clinical prediction models consisting of risk factors seem to be equivalent to OGTT in the prediction of future type 2 diabetes in younger subjects at short follow-up, but not in older subjects or during longer follow-up (6). To our knowledge, however, only very few studies have explored these relationships at follow-up beyond 10 years.

Furthermore, previous studies including the Malmö Preventive Project (MPP) and the Botnia Study have demonstrated that glucose obtained at 60 min during OGTT may be a superior predictor of the incident type 2 diabetes when compared with both the traditionally used 120 min glucose measurement as well as a clinical prediction model without FPG (7). In addition, it was indicated that adding early OGTT-derived blood glucose (BG) measurements to clinical risk factors may provide incremental predictive benefit in specific populations and during certain circumstances. Finally, the predictive values of all models were lower in MPP, except for FPG, suggesting that the differences in predictive properties might not be only due to the differences in follow-up duration (23.5 years in MPP vs 4.94 years in the Botnia Study). This study was undertaken with the following purposes: (i) To evaluate whether the addition of OGTT provides incremental prognostic value on top of clinical risk factors, including FBG, for the prediction of type 2 diabetes; (ii) to assess whether BG measured at an earlier time point during OGTT provides better prognostic yield than BG measured at $120 \mathrm{~min}$; (iii) to explore whether the prognostic properties of these prediction models vary according to the follow-up time.

\section{Subjects and methods}

\section{Study population}

The Malmö Preventive Project (1974-1992, $n=33346)$ was a population-based cohort study conducted among the inhabitants in Malmö, Sweden, belonging to prespecified birth cohorts between 1921 and 1949 (8). Progressively older subjects were recruited later during the inclusion process. A total of 22444 males and 10902 females attended the screening program, with an overall attendance rate of $71 \%$ ( $75 \%$ for men). All subjects answered a self-administered questionnaire on lifestyle, family history of diabetes (first-degree relatives) and cardiovascular disease, medical history, and current medication. Current smoking was defined as smoking at least one cigarette a day, and sedentary lifestyle was defined as leisure time mostly spent on sedentary activities. Prevalent diabetes was defined as self-reported diabetes or according to the 1985 WHO criteria $(9,10)$. Height and weight in light indoor clothing were measured, from which body mass index (BMI) was calculated. Blood pressure was measured twice in the right arm after 10 min of supine rest, with the mean value recorded for analysis. Moreover, blood samples were obtained after $\geq 10 \mathrm{~h}$ overnight fasting for measurement of BG, plasma insulin, serum lipids, and serum creatinine. BG was analyzed by the glucoseoxidase method (1974-1977) or the hexokinase method (1977-1992) (11). The serum samples were analyzed using the laboratory's standard methods. Plasma insulin was analyzed by a standard radioimmunoassay method (12). MPP was conducted in accordance with the Declaration of Helsinki.

\section{Oral glucose tolerance test}

In 18960 participants without prevalent diabetes, a $120 \mathrm{~min}$ OGTT was performed by ingestion of $30 \mathrm{~g}$ glucose per $\mathrm{m}^{2}$ body surface area (BSA; DuBois equation) in a $10 \%$ aqueous solution within $5 \mathrm{~min}$. BG levels were determined at $0,20,40,60,90$, and $120 \mathrm{~min}$.

\section{Recording of events}

The follow-up time for each subject was defined as time elapsed from baseline screening to the date of type 2 diabetes diagnosis (type 2 diabetes event), death, emigration, or last follow-up date, although no more than 30 years. Type 2 diabetes events were recorded by 14 national and local registries and comprised International Classification of Diseases (ICD-10) codes E11.0-E11.9. The method to ascertain type 2 diabetes diagnosis in the Swedish Inpatient Register has been previously validated (13). Mortality follow-up was based on the national registry on causes of mortality at the Central Bureau of Statistics, Sweden.

\section{Final study population}

Subjects with a missing BG value $(n=27728)$ were excluded from this study. By far, most subjects did not undergo BG measurements at 20,40,60, and/or 90 min during OGTT, which was due to financial issues. This was especially 
evident for the female study subjects as they were mostly screened during the latter half of the inclusion period, i.e. 1981-1992. The remaining 132 females with BG measurements at these intermediate time points during OGTT were therefore excluded as well. The remaining subjects with missing information regarding family history of diabetes $(n=26)$, other baseline variables $(n=23)$, or incomplete follow-up due to emigration $(n=70)$ were likewise excluded. Of the 5367 subjects left, 111 had prevalent diabetes, leaving a final study population comprising 5256 males (Fig. 1). The subjects receiving cardiovascular medication were kept in the study, as they comprised less than $5 \%$ of the study population, and their exclusion did not significantly alter the study results.

\section{Statistical analysis}

Continuous variables were summarized by means and standard deviations (normally distributed variables) and medians and interquartile ranges (IQR; non-normally distributed variables), whereas categorical variables were presented by frequencies and corresponding percentages. Groupwise comparisons were performed using independent samples $t$-test, Mann-Whitney $U$-test, and Pearson's $c^{2}$-test. A simple multivariable Cox proportional hazards regression model (clinical prediction model) for predicting incident type 2 diabetes was derived using a panel of clinically important and statistically significant baseline characteristics as the independent variables, and type 2 diabetes events recorded until 30 years of follow-up or censoring. Furthermore, model

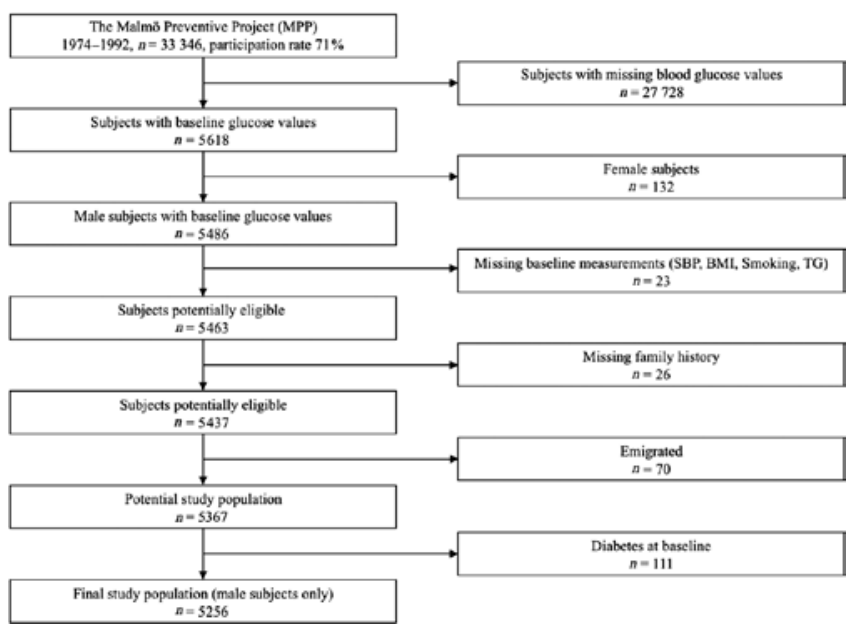

Figure 1

Flowchart depicting the selection of the study population. SBP, systolic blood pressure; TG, triglycerides. discrimination was assessed using Harrell's concordance index (C-index) (14) with all calculations repeated using cumulated events $(5,10,20$, and 30 years of follow-up). The number of subjects still at risk at each of these time points was 5103, 4806, 3997, and 2850 respectively. Since the last subject was included on November 2nd 1983, complete 30-year follow-up data were available for all subjects. In addition, the incremental predictive value of each BG measurement during OGTT was evaluated using integrated discrimination improvement (IDI) (15). The analyses were focused on BG measured at $60 \mathrm{~min}, 90 \mathrm{~min}$, and $120 \mathrm{~min}$ because of the better overall predictive capacity compared with BG measured at 20 and $40 \mathrm{~min}$ (analyses available upon request). Since the distributions of serum triglycerides (TG) and creatinine were moderately positively skewed, they were both natural log-transformed for the regression analyses. All analyses were carried out using IBM SPSS Statistics 23 (IBM) and Stata/IC 14 (StataCorp LP, College Station, TX, USA).

\section{Results}

\section{Characteristics of the study population}

At baseline, subjects had a median age of 48 (48-49) years, mean BP $131 \pm 16 \mathrm{mmHg}$ systolic and $88 \pm 11 \mathrm{mmHg}$ diastolic, and mean BMI $24.9 \pm 3.3 \mathrm{~kg} / \mathrm{m}^{2}$. Total cholesterol was $5.8 \pm 1.0 \mathrm{mmol} / \mathrm{L}$. During follow-up (median 30 (20-30) years), 676 incident cases of type 2 diabetes were detected, corresponding to 5.3 cases per 1000 person-years. The subjects who developed type 2 diabetes had significantly greater baseline BMI, systolic BP (SBP), diastolic BP (DBP), total cholesterol, TG, FBG, and BG values at 20,40,60, 90, and 120 min (Table 1). Peak BG levels were achieved at $40 \mathrm{~min}$. There were no significant between-group differences regarding smoking status and sedentary lifestyle.

\section{Fasting blood glucose and the clinical prediction model}

Using Cox regression modeling at 30-year follow-up, the following variables were statistically significant on univariable analysis for prediction of incident type 2 diabetes: BMI, SBP, DBP, FBG, total cholesterol, TG, sedentary lifestyle, and family history of diabetes. The initial clinical model included all of these except SBP, since SBP and DBP displayed significant colinearity; however, with DBP performing better than SBP. Total cholesterol 
Table 1 Baseline characteristics in subjects with and without incident type 2 diabetes detected at follow-up. Categorical variables are given as $n(\%)$, whereas continuous variables are given as mean \pm s.D. (approximately normally distributed variables) or median (IQR) (non-normally distributed variables).

\begin{tabular}{|c|c|c|}
\hline Variable & $\begin{array}{l}\text { All subjects } \\
(n=5256)\end{array}$ & $\begin{array}{l}\text { No incident type } 2 \text { diabetes } \\
(n=4580)\end{array}$ \\
\hline Age (years) & $48(48-49)$ & $48(48-49)$ \\
\hline Family history of diabetes & $703(13 \%)$ & $553(12 \%)$ \\
\hline Sedentary lifestyle & $3005(57 \%)$ & $2597(57 \%)$ \\
\hline Active smoking & $2754(52 \%)$ & $2408(53 \%)$ \\
\hline BMI $\left(k g / m^{2}\right)$ & $24.9 \pm 3.3$ & $24.6 \pm 3.2$ \\
\hline Systolic BP (mmHg) & $131 \pm 16$ & $130 \pm 16$ \\
\hline Diastolic BP $(\mathrm{mmHg})$ & $88 \pm 11$ & $88 \pm 10$ \\
\hline Fasting blood glucose (mmol/L) & $4.7 \pm 0.6$ & $4.6 \pm 0.5$ \\
\hline Blood glucose at $20 \mathrm{~min}(\mathrm{mmol} / \mathrm{L})$ & $7.6 \pm 1.4$ & $7.6 \pm 1.4$ \\
\hline Blood glucose at $40 \mathrm{~min}(\mathrm{mmol} / \mathrm{L})$ & $9.1 \pm 1.8$ & $8.9 \pm 1.8$ \\
\hline Blood glucose at $60 \mathrm{~min}(\mathrm{mmol} / \mathrm{L})$ & $8.1 \pm 2.1$ & $7.9 \pm 2.0$ \\
\hline Blood glucose at $90 \mathrm{~min}(\mathrm{mmol} / \mathrm{L})$ & $6.6 \pm 1.7$ & $6.4 \pm 1.6$ \\
\hline Blood glucose at $120 \mathrm{~min}(\mathrm{mmol} / \mathrm{L})$ & $5.4 \pm 1.5$ & $5.3 \pm 1.4$ \\
\hline Total cholesterol (mmol/L) & $5.8 \pm 1.0$ & $5.8 \pm 1.0$ \\
\hline Triglycerides $(\mathrm{mmol} / \mathrm{L})$ & $1.4(1.1-1.9)$ & $1.4(1.0-1.8)$ \\
\hline Creatinine $(\mu \mathrm{mol} / \mathrm{L})$ & $94(86-102)$ & $94(86-102)$ \\
\hline Follow-up time (years) & $30(20-30)$ & $30(22-30)$ \\
\hline
\end{tabular}

\begin{tabular}{l}
$\begin{array}{l}\text { Incident type } 2 \text { diabetes } \\
(n=676)\end{array}$ \\
\hline $48(48-49)$ \\
$150(22 \%)$ \\
$408(60 \%)$ \\
$346(51 \%)$ \\
$26.6 \pm 3.4$ \\
$134 \pm 17$ \\
$91 \pm 11$ \\
$4.9 \pm 0.6$ \\
$8.0 \pm 1.4$ \\
$10.0 \pm 1.7$ \\
$9.4 \pm 2.0$ \\
$7.6 \pm 1.9$ \\
$6.1 \pm 1.6$ \\
$5.9 \pm 1.0$ \\
$1.7(1.3-2.3)$ \\
$94(86-102)$ \\
$21(15-26)$ \\
\hline
\end{tabular}

\begin{tabular}{c}
\hline P-values \\
\hline $0.002^{\star}$ \\
$<0.0001^{\dagger}$ \\
$0.07^{\dagger}$ \\
$0.50^{\dagger}$ \\
$<0.0001^{\ddagger}$ \\
$<0.0001^{\ddagger}$ \\
$<0.0001^{\ddagger}$ \\
$<0.0001^{\ddagger}$ \\
$<0.0001^{\ddagger}$ \\
$<0.0001^{\ddagger}$ \\
$<0.0001^{\ddagger}$ \\
$<0.0001^{\ddagger}$ \\
$<0.0001^{\ddagger}$ \\
$0.049^{\ddagger}$ \\
$<0.0001^{\star}$ \\
$0.59^{*}$ \\
$<0.0001^{\star}$
\end{tabular}

${ }^{*}$ Mann-Whitney $U$ test; ${ }^{\dagger}$ Pearson's $c^{2}$-test; ${ }^{*}$ independent samples $t$-test.

and sedentary lifestyle did not result in significant model improvement and were excluded. Age was forced into the model, as older subjects on average had shorter follow-up due to the inclusion process. Therefore, the final optimized clinical Cox regression model included age, BMI, DBP, FBG, TG, and family history of diabetes (clinical prediction model, likelihood-ratio $c^{2}=449.44$ ). Supplementary analyses using type 2 diabetes events at shorter time points revealed that the statistically optimized clinical prediction model would include fewer variables at the two shortest pre-specified follow-up periods, i.e. age, FBG, and TG at 5 years (Cox regression likelihood-ratio $c^{2}=57.00$; C-index: 0.821), and age, BMI, FBG, TG, and family history at 10 years (Cox regression likelihood-ratio $c^{2}=116.10$; C-index: 0.764). However, all the following analyses were based on the model derived using 30-year follow-up (Table 2).

Table 2 Cox proportional hazards regression model (clinical prediction model) for the prediction of type 2 diabetes.

\begin{tabular}{|c|c|c|}
\hline Variable & Hazard ratio $(95 \% \mathrm{Cl})$ & $P$-value \\
\hline Age (years) & $1.02(0.98-1.06)$ & 0.25 \\
\hline BMI $\left(\mathrm{kg} / \mathrm{m}^{2}\right)$ & $1.14(1.11-1.16)$ & $<0.0001$ \\
\hline $\mathrm{DBP}(\mathrm{mmHg})$ & $1.01(1.002-1.02)$ & 0.01 \\
\hline FBG (mmol/L) & $1.98(1.72-2.28)$ & $<0.0001$ \\
\hline $\mathrm{TG}(\mathrm{mmol} / \mathrm{L}) *$ & $1.66(1.40-1.96)$ & $<0.0001$ \\
\hline Family history of diabetes & $1.84(1.53-2.21)$ & $<0.0001$ \\
\hline
\end{tabular}

Likelihood-ratio $c^{2}=449.44 ;$ *After logarithmic transformation.

\section{Oral glucose tolerance test}

Both $60\left(\mathrm{OGTT}_{60}\right)$ and 90 min BG $\left(\mathrm{OGTT}_{90}\right)$ consistently performed better than 120 min BG $\left(\right.$ OGTT $\left._{120}\right)(P \geq 0.10$ at 5 years, $P \leq 0.04$ at 10,20 , and 30 years). There were no significant differences in C-index between OGTT $_{60}$ and $\mathrm{OGTT}_{90}$, regardless of follow-up duration. The predictive capacity (i.e. C-index) declined with increasing follow-up time. In addition, when comparing OGTT alone with the clinical prediction model, the predictive capacities were similar at 5 years $(P \geq 0.40$ for all comparisons); however, at 10 years, OGTT $_{60}$ and OGTT $_{90}$, but not OGTT 120 performed significantly better than the clinical model $\left(P=0.02\right.$ for OGTT $_{60}, P=0.04$ for OGTT $_{90}$, and $P=0.58$ for OGTT $_{120}$, respectively). Beyond 10 years, the clinical model performed increasingly better than OGTT, although nonsignificantly for most comparisons (Table 3).

\section{Multivariable prediction models with oral glucose tolerance test}

The multivariable prediction models were constructed by adding a single BG obtained during OGTT, i.e. BG at 60,90 , or $120 \mathrm{~min}$, to the clinical prediction model. All these multivariable models, regardless of follow-up duration, performed significantly better than the clinical model ( $P \leq 0.045$ for all comparisons), except the multivariable model incorporating OGTT $_{120}$ for 5-year risk prediction $(P=0.08)$. With increasing 
follow-up time, C-index decreased in all models, although mostly in the multivariable models (Table 3). Supporting the influence of follow-up duration on the additive predictive value of OGTT, only OGTT $_{120}$ was associated with a significant IDI at 5 years $(P=0.01)$, whereas OGTT $_{60}$, OGTT $_{90}$, and OGTT $_{120}$ displayed a significant IDI at 10 years and beyond $(P \leq 0.0001$ for all comparisons) (Table 4).

Table 3 Harrell's concordance index (C-index) for each prediction model, and $P$-values for between-model comparisons.

Incident type 2 diabetes at 5 years

\begin{tabular}{c}
\hline C-index \\
\hline \\
\\
0.805 \\
0.838 \\
0.866 \\
0.883 \\
0.846 \\
0.884 \\
0.909 \\
0.885 \\
0.913 \\
0.904 \\
0.903
\end{tabular}

Incident type 2 diabetes at 10 years

\section{FBG}

Clinical

OGTT $_{60}$

OGTT $_{90}$

OGTT $_{120}$

Multi $_{60}$

Multi $_{90}$

Multi $_{120}$

Multi $60+90$

Multi $_{60+120}$

Multi $_{90+120}$

Incident type $\mathbf{2}$ diabetes at $\mathbf{2 0}$ years

FBG

Clinical

OGTT $_{60}$

$\mathrm{OGTT}_{90}$

OGTT $_{120}$

Multi $_{60}$

Multi $_{90}$

Multi $_{120}$

Multi $_{60+90}$

Multi $_{60+120}$

Multi $_{90+120}$

Incident type $\mathbf{2}$ diabetes at $\mathbf{3 0}$ years

FBG

Clinical

OGTT $_{60}$

$\mathrm{OGTT}_{90}$

OGTT $_{120}$

Multi $_{60}$

Multi $_{90}$

Multi $_{120}$

Multi $60+90$

Multi $_{60+120}$

Multi $_{90+120}$

\begin{tabular}{lll}
\hline $\mathbf{O G T T}_{\mathbf{6 0}}$ & $\mathbf{O G T T}_{\mathbf{9 0}}$ \\
\cline { 1 - 1 } 0.26 & & 0.16 \\
0.59 & & 0.40 \\
& & 0.48 \\
&
\end{tabular}

0.717

0.765

0.832

0.831

0.783

0.824

0.850

0.821

0.856

0.848

0.849

0.690

0.774

0.778

0.764

0.709

0.808

0.809

0.792

0.818

0.814

0.809

$\begin{array}{ccc}<0.0001 & 0.001 & 0.06 \\ 0.02 & 0.04 & 0.58 \\ & 0.95 & 0.04 \\ & & 0.005\end{array}$

$$
\begin{array}{cc}
<0.0001 & <0.0001 \\
0.82 & 0.29 \\
& 0.17
\end{array}
$$

0.648

0.732

0.731

0.713

0.662

0.764

0.760

0.743

0.770

0.768

0.760

0.06

0.005

0.4

$<0.0001$

$<0.0001$

0.231

$<0.0001$

\begin{tabular}{c}
\hline OGTT $_{\mathbf{1 2 0}}$ \\
\hline \\
\\
0.42 \\
0.87 \\
0.60 \\
0.10
\end{tabular}

\begin{tabular}{c} 
Multi $_{60}$ \\
\hline 0.006
\end{tabular}

$0.30 \quad 0.96$
0.15

$\begin{array}{lll}0.23 & 0.35 & \\ 0.32 & & 0.10 \\ & 0.25 & 0.15\end{array}$

$\begin{array}{llll}0.58<0.0001 & <0.0001 & 0.004\end{array}$

$0.10 \quad 0.83$

0.02

$\begin{array}{ccc}0.04 & 0.02 & \\ 0.09 & & <0.0001 \\ & 0.50 & 0.01\end{array}$

$\begin{array}{llll}<0.0001 & <0.0001 & 0.001 & 0.02\end{array}$

$\begin{array}{ll}0.99 & 0.03 \\ & 0.01\end{array}$

$0.12<0.0001$

$0.28<0.0001$

$0.53 \quad 0.01$

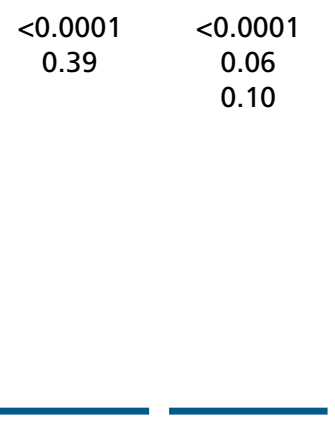

$<0.0001<0.0001<0.0001 \quad 0.008$

$<0.0001$

$0.45 \quad 0.002$

$0.15<0.0001$

$0.17<0.0001$

$0.84 \quad 0.006$

FBG, fasting blood glucose; Clinical, clinical prediction model (age, BMI, diastolic BP, fasting blood glucose, triglycerides, family history of diabetes); $\mathrm{OGTT}_{60}$, blood glucose measured at $60 \mathrm{~min}$ during oral glucose tolerance test; Multi ${ }_{60}$, multivariable prediction model (clinical prediction model + blood glucose measured at 60 min during oral glucose tolerance test). 
Table 4 Absolute integrated discrimination improvement for the various multivariable prediction models (including $P$-values) at $5,10,20$, and 30 years.

\begin{tabular}{|c|c|c|c|c|}
\hline & 5 years & 10 years & 20 years & 30 years \\
\hline Multi $_{60}$ vs clinical & $0.006(0.11)$ & $0.015(0.0001)$ & $0.0334(<0.0001)$ & $0.0306(<0.0001)$ \\
\hline Multi ${ }_{90}$ vs clinical & $0.017(0.057)$ & $0.0447(<0.0001)$ & $0.0391(<0.0001)$ & $0.0301(<0.0001)$ \\
\hline Multi $_{120}$ vs clinical & $0.018(0.01)$ & $0.0328(<0.0001)$ & $0.0212(<0.0001)$ & $0.0159(<0.0001)$ \\
\hline Multi $_{60+90}$ vs Multi $_{60}$ & $0.1175(0.81)$ & $0.02854(<0.0001)$ & $0.01027(<0.0001)$ & $0.00496(0.0001)$ \\
\hline Multi ${ }_{60+120}$ vs Multi $i_{60}$ & $0.01508(0.03)$ & $0.02513(<0.0001)$ & $0.00900(0.0005)$ & $0.00539(0.0001)$ \\
\hline Multi $_{90+60}$ vs Multi $_{90}$ & $0.0003(0.77)$ & $-0.00102(0.44)$ & $0.00454(0.007)$ & $0.00548(<0.0001)$ \\
\hline Multi $_{90+120}$ vs Multi ${ }_{90}$ & $0.00418(0.22)$ & $0.00258(0.11)$ & $0.00023(0.42)$ & $0.00012(0.57)$ \\
\hline Multi $_{120+60}$ vs Multi $_{120}$ & $0.00278(0.52)$ & $0.00745(0.03)$ & $0.02120(<0.0001)$ & $0.02008(<0.0001)$ \\
\hline Multi $_{120+90}$ vs Multi $_{120}$ & $0.00336(0.55)$ & $0.01446(0.004)$ & $0.01816(<0.0001)$ & $0.01428(<0.0001)$ \\
\hline
\end{tabular}

Clinical, clinical prediction model (age, BMI, diastolic BP, fasting blood glucose, triglycerides, family history of diabetes); Multi ${ }_{60}$, multivariable prediction model (clinical prediction model + blood glucose measured at 60 min during oral glucose tolerance test).

Addition of another OGTT-derived BG to the above did not improve the predictive value any further, except at 10 years, for which a subtle, but statistically significant benefit was observed for the combination of the clinical prediction model and both OGTT $_{60}$ and OGTT $_{90}$ (Table 3). Consistent with the high sensitivity of the IDI method, significant additional effects were noted for most combinations of two OGTT-derived BG beyond 5 years, although the numerical increases were minimal (Table 4).

Multivariable models including either OGTT $_{60}$ or OGTT $_{90}$ outperformed the multivariable model with OGTT $_{120}$ at more than 10 and 5 years of follow-up respectively, whereas the differences between multivariable models with OGTT $_{60}$ and OGTT 90 were subtle (Table 3).

\section{Discussion}

Concordant with our hypotheses, the main findings of our study were: (i) Addition of OGTT to a clinical prediction model including FBG provided incremental prognostic value for the prediction of type 2 diabetes; (ii) BG measured at 60 and 90 min during OGTT provided better prognostic yield than BG measured at $120 \mathrm{~min}$, both isolated and in addition to the clinical prediction model, at follow-up longer than 10 and 5 years respectively; and (iii) The performance of all prediction models decreased over time, especially evident for models with OGTT $_{120}$ and less so for models without OGTT.

Several prediction models for incident type 2 diabetes based on clinical risk factors have been suggested $(6,16,17)$ because of the ease of administration, convenience, acceptability to patients, and lower cost in comparison with OGTT. Data from The San Antonio Heart Study demonstrated that a simple clinical prediction model resulted in better identification of subjects at risk than OGTT during a 7- to 8-year follow-up period (16). In contrast, although in accordance with our results, McNeely et al. (6) found no significant difference between a clinical model and OGTT for the 5- to 6-year prediction of type 2 diabetes in subjects aged $\leq 55$ years, whereas Alyass et al. (7) found a superior discrimination ability of OGTT; however, their clinical prediction model did not include FPG, which by itself had an area under the receiver-operating characteristics curve comparable with OGTT $_{120}$, but lower than OGTT $_{60}$. One possible explanation for the heterogeneous results between studies could be the use of different diagnostic criteria for type 2 diabetes. For instance, one would assume a relatively better prognostic yield of OGTT if more incident type 2 diabetes cases were diagnosed according to OGTT criteria. As the diagnostic threshold for FPG was lowered in 1997, relatively more subjects received a diagnosis of diabetes based on the OGTT criteria before 1997, during which most cases in this study were identified. This may explain why OGTT performed well in our study, especially at a short-term follow-up (18).

As a substantial number of subjects who eventually develop type 2 diabetes have normal glucose tolerance (NGT) and/or normal fasting glucose at baseline, and IGT and impaired fasting glucose (IFG) display very limited overlap $(19,20,21)$, the combination of a clinical prediction model with OGTT may potentially identify a larger percentage of high risk subjects. However, Stern et al. (16) found only a minor improvement, and Wilson et al. (22) did not find additional capability to discriminate persons who developed type 2 diabetes from those who did not, when adding OGTT $_{120}$ to a clinical model. Possible explanations may be the use of a $75 \mathrm{~g}$ glucose loading dose, shorter follow-up duration (7.5 years and 7 years, respectively), subjects with greater mean BMI $\left(27.1 \mathrm{~kg} / \mathrm{m}^{2}\right.$ in both studies), the inclusion of both male and female subjects, and the use of clinical prediction models with 
a larger number of co-variables, many of which were not significantly associated with the outcome in multivariable analysis. In this study, addition of any one OGTT-derived BG to the clinical prediction model resulted in significant model improvement for most comparisons, but the benefit of further adding a second OGTT-derived BG was very limited, i.e. a single measurement may be a reliable indicator of glucose tolerance, at least for prediction purposes.

\section{Timing of glucose measurements during the oral glucose tolerance test}

The cut-offs for glucose intolerance and diabetes after glucose loading in non-pregnant subjects have traditionally been based on OGTT $_{120}(23,24)$. However, this topic has been debated for at least 40 years (23), and a growing body of evidence supports the use of OGTT $_{60}$. In addition to the study by Alyass et al. (7) which demonstrated better capability of OGTT ${ }_{60}$ compared with OGTT $_{120}$, both isolated and combined with other clinical risk factors, for predicting incident type 2 diabetes at 4.94 and 23.5 years, authors Abdul-Ghani et al. $(21,25$, $26,27,28)$ showed that OGTT $_{60}$ performed superiorly to OGTT $_{120}$ for identifying subjects at high risk of developing type 2 diabetes, and that addition of OGTT $_{60}$ to multivariable prediction models resulted in significant model improvement similar to adding OGTT $_{120}$, during a 7 to 8-year follow-up period. Likewise, Kuang et al. (29) demonstrated a better predictive ability of OGTT $_{60}$ compared with OGTT $_{120}$ at 10-year follow-up. In this study, both OGTT $_{60}$ and OGTT 90 performed significantly better than OGTT $_{120}$ for most comparisons, including the multivariable models. Lastly, Janghorbani et al. (30) found almost equal discriminating abilities of OGTT $_{60}$ and OGTT $_{120}$ during a follow-up time of 3-5 years. However, the study population was highly selected and included only first-degree relatives of patients with type 2 diabetes. The generally superior predictive ability of BG obtained at earlier time points during OGTT may reflect the crucial role of the first-phase insulin response in postprandial glucose homeostasis $(31,32)$.

\section{Importance of follow-up duration}

Most previous similar studies have not assessed the predictive value of clinical models with additional OGTT beyond 10 years of follow-up. Furthermore, the impact of follow-up duration on the discriminating ability of prediction models has not been well studied. Nonetheless,
McNeely et al. (6) found no significant difference between a clinical model and OGTT $_{120}$ for neither 5 to 6 -year nor 10-year prediction of type 2 diabetes in subjects $\leq 55$ years. In this study, the predictive capacity of all models decreased, when follow-up increased. This is not surprising since at least some of the risk factors are modifiable and show considerable changes over time (33). The fact that the clinical prediction model performed better than OGTT alone beyond 10 years may therefore simply be the inclusion of non-modifiable risk factors, which also explains why the relative incremental predictive ability of OGTT decreased with increasing follow-up time. Also, a substantial proportion of individuals with IGT may revert to NGT on a subsequent OGTT (34). Finally, the clinical model included several components of the metabolic syndrome and may identify subjects who are more prone to develop diabetes in the long-term, even before demonstrating elevated BG (35), which was supported by the fact that the optimal clinical prediction model at short-term follow-up mainly included FBG. Beyond the 5-year-mark, OGTT $_{60}$ and OGTT $_{90}$ were consistently significantly better predictors than OGTT $_{120}$. The multivariable models with OGTT $_{60}$ or OGTT $_{90}$ displayed better predictive properties than the clinical models at all time points. However, IDI was greatest for the multivariable model with OGTT $_{120}$ at 5 -year follow-up, but was surpassed by the multivariable model with OGTT $_{90}$ at 10-year follow-up and by the multivariable model with OGTT $_{60}$ at 20-year follow-up, whereby one might speculate that BG obtained at earlier time points during OGTT particularly predicts late incident type 2 diabetes, whereas OGTT $_{120}$ predicts early incident type 2 diabetes. This may additionally explain the controversies concerning the optimal timing of BG measurement during OGTT.

\section{Limitations}

In the last few decades, there has been a number of changes in the definition of type 2 diabetes, e.g. the lowering of the diagnostic threshold for FPG-defined diabetes in 1997 and the introduction of $\mathrm{HbA}_{1 \mathrm{c}}$-defined diabetes in 2011 (18, http://www.who.int/diabetes/publications/diagnosis_diabetes2011/en/ (cited 2015 July 20)). The subjects defined according to these different measures only partially overlap, both with respect to pre-diabetes and diabetes $(36,37)$. However, the predictive capability of risk factors associated with incident type 2 diabetes defined by either FPG or $\mathrm{HbA}_{1 \mathrm{c}}$ seems comparable (38). The addition of $\mathrm{HbA}_{1 \mathrm{c}}$ to our study would have been desirable, but these 
measurements were not done. Furthermore, at the time of inclusion, a standard OGTT comprised a $30 \mathrm{~g} / \mathrm{m}^{2}$ glucose load which, however, seems to yield results similar to the $75 \mathrm{~g}$ load (39). Our exclusion of a significant proportion of the original study population, although based on financial reasons, constitutes a major limitation and prevents us from extending our results beyond middle-aged Caucasian men, and due to the large decline in subjects at risk in the latter half of the study, the proportional hazards assumption assessed using Schoenfeld residuals was violated at 20- and 30-year follow-up. Still, the choice of comparing C-indices derived from Cox models was superior to comparing areas under the curves derived from logistic models, and additional analyses revealed the same patterns for OGTT when using a composite endpoint comprising diabetes and death. Furthermore, we found no significant interaction between OGTT and death. It should also be remembered that prediction models generally tend to overestimate the actual event risk (5). Lastly, differences between-study results might further be due to variations in sex ratio, ethnicity, sample sizes, inclusion criteria, co-variables included, glucose load used for OGTT, and follow-up duration.

\section{Clinical perspectives}

Identifying subjects at an increased risk for future diabetes has become more relevant because of the proven preventive effects of lifestyle modification and medication in subjects with IGT $(2,3,4)$. Our findings may carry future clinical implications, as shorter examination time is associated with lower cost and greater convenience for both patients and health care professionals. Although the introduction of $\mathrm{HbA}_{\mathrm{c}}$ as a diagnostic tool for diabetes has led to fewer OGTTs in general, it is well known that these methods do not always identify diabetes in the same subjects $(36,37)$. Therefore, a role for OGTT likely exists in subjects with a high normal or an only slightly elevated $\mathrm{HbA}_{c}$, in order to reach a definitive diagnostic decision. Supplementary analyses evaluating the role of OGTT in subjects with and without IFG, defined according to the current World Health Organization and American Diabetes Association criteria, revealed no significant interaction for the predictive value of OGTT between subjects with NFG and IFG, respectively. This indicates that our findings in the entire study population are also applicable to the subgroup of patients with IFG who to some degree may reflect the subjects with borderline HbA1c, i.e. subjects in whom an OGTT could be considered in daily clinical practice. However, our findings need further replication and external validation. In addition, interventional studies are necessary in order to prove the benefit of secondary prevention in the subjects found through multivariable screening, including the use of OGTT $_{60}$ or OGTT ${ }_{90}$, and not only those with IGT defined by OGTT 120 .

Such trials are also prudent in order to address the real magnitude of effect of adding screening with the OGTT to the other risk factors in the relevant clinical setting.

In conclusion, in this prospective population-based cohort study, we found that the addition of an OGTTderived $\mathrm{BG}$ to a clinical prediction model provided incremental prognostic value, including IDI, in the prediction of type 2 diabetes, but relatively less so at longer follow-up. BG measured at 60 or 90 min during OGTT provided better prognostic yield than BG measured at $120 \mathrm{~min}$ at follow-up longer than 10 and 5 years respectively.

\section{Declaration of interest}

The authors declare that there is no conflict of interest that could be perceived as prejudicing the impartiality of the research reported.

\section{Funding}

This study was funded by a research grant from The Heart and Lung Foundation of Sweden and The Danish Diabetes Academy supported by the Novo Nordisk Foundation.

\section{Author contribution statement}

Mette Lundgren Nielsen, Manan Pareek and Michael Hecht Olsen contributed substantially to the conception of the hypothesis, the design of the work, analysis of the work, interpretation of data for the work, drafting the work. Margrét Leósdóttir, Karl-Fredrik Eriksson and Peter $\mathrm{M}$ Nilsson contributed substantially to the acquisition of data for the work whilst Kurt Højlund contributed substantially to the interpretation of data for the work. All authors contributed equally to critical revision for important intellectual content, final approval of the version to be published, and agrees to be accountable for all aspects of the work in ensuring that questions related to the accuracy or integrity of any part of the work are appropriately investigated and resolved.

All authors take responsibility for all aspects of the reliability and freedom from bias of the data presented and their discussed interpretation.

\section{References}

1 Wang YC, McPherson K, Marsh T, Gortmaker SL \& Brown M. Health and economic burden of the projected obesity trends in the USA and the UK. Lancet 2011378 815-825. (doi:10.1016/S01406736(11)60814-3)

2 Tuomilehto J, Lindstrom J, Eriksson JG, Valle TT, Hamalainen H, Ilanne-Parikka P, Keinanen-Kiukaanniemi S, Laakso M, Louheranta A, Rastas $\mathrm{M}$ et al. Prevention of type 2 diabetes mellitus by changes in 
lifestyle among subjects with impaired glucose tolerance. New England Journal of Medicine 2001344 1343-1350.

(doi:10.1056/NEJM200105033441801)

3 Knowler WC, Barrett-Connor E, Fowler SE, Hamman RF, Lachin JM, Walker EA, Nathan DM \& Diabetes Prevention Program Research Group. Reduction in the incidence of type 2 diabetes with lifestyle intervention or metformin. New England Journal of Medicine 2002346 393-403. (doi:10.1111/ dme.2012.30.issue-1)

4 Chiasson JL, Josse RG, Gomis R, Hanefeld M, Karasik A, Laakso M \& Stop-Niddm Trail Research Group. Acarbose for prevention of type 2 diabetes mellitus: the STOP-NIDDM randomised trial. Lancet 2002 359 2072-2077.

5 Abbasi A, Peelen LM, Corpeleijn E, van der Schouw YT, Stolk RP, Spijkerman AM, Van der A Dl, Moons KG, Navis G, Bakker SJ et al. Prediction models for risk of developing type 2 diabetes: systematic literature search and independent external validation study. The British Medical Journal 2012345 e5900. (doi:10.1136/bmj.e5900)

6 McNeely MJ, Boyko EJ, Leonetti DL, Kahn SE \& Fujimoto WY. Comparison of a clinical model, the oral glucose tolerance test, and fasting glucose for prediction of type 2 diabetes risk in Japanese Americans. Diabetes Care 200326 758-763. (doi:10.2337/dc15-0625)

7 Alyass A, Almgren P, Akerlund M, Dushoff J, Isomaa B, Nilsson P, Tuomi T, Lyssenko V, Groop L, Meyre D et al. Modelling of OGTT curve identifies $1 \mathrm{~h}$ plasma glucose level as a strong predictor of incident type 2 diabetes: results from two prospective cohorts. Diabetologia 201558 87-97. (doi:10.1007/s00125-014-3390-x)

8 Berglund G, Eriksson KF, Israelsson B, Kjellstrom T, Lindgarde F, Mattiasson I, Nilsson JA \& Stavenow L. Cardiovascular risk groups and mortality in an urban swedish male population: the Malmo Preventive Project. Journal of Internal Medicine 1996239 489-497. (doi:10.1046/j.1365-2796.1996.483819000.x)

9 Diabetes mellitus. Report of a WHO Study Group. World Health Organization Technical Report Series 1985727 1-113.

10 Eriksson KF \& Lindgarde F. Impaired glucose tolerance in a middle-aged male urban population: a new approach for identifying high-risk cases. Diabetologia 199033 526-531.

11 Carroll JJ, Smith N \& Babson AL. A colorimetric serum glucose determination using hexokinase and glucose-6-phosphate dehydrogenase. Biochemical Medicine 19704 171-180. (doi:10.1016/0006-2944(70)90093-1)

12 Nilsson P, Nilsson JA, Hedblad B, Eriksson KF \& Berglund G. Hyperinsulinaemia as long-term predictor of death and ischaemic heart disease in nondiabetic men: The Malmo Preventive Project. Journal of Internal Medicine 2003253 136-145. (doi:10.1046/j.1365-2796.2003.01064.x)

13 Ludvigsson JF, Andersson E, Ekbom A, Feychting M, Kim JL, Reuterwall C, Heurgren M \& Olausson PO. External review and validation of the Swedish national inpatient register. BMC Public Health 201111 450. (doi:10.1186/1471-2458-11-450)

14 Harrell FE Jr, Califf RM, Pryor DB, Lee KL \& Rosati RA. Evaluating the yield of medical tests. Journal of the American Medical Association 1982247 2543-2546. (doi:10.7326/0003-4819-118-2199301150-00001)

15 Pencina MJ, D’Agostino RB Sr, D,Agostino RB Jr \& Vasan RS. Evaluating the added predictive ability of a new marker: from area under the ROC curve to reclassification and beyond. Statistics in Medicine 200827 157-172 (discussion 207-212).

16 Stern MP, Williams K \& Haffner SM. Identification of persons at high risk for type 2 diabetes mellitus: do we need the oral glucose tolerance test? Annals of Internal Medicine 2002136 575-581. (doi:10.1016/j.metabol.2009.04.030)
17 Lindstrom J \& Tuomilehto J. The diabetes risk score: a practical tool to predict type 2 diabetes risk. Diabetes Care 200326 725-731.

18 Report of the Expert Committee on the Diagnosis and Classification of Diabetes Mellitus. Diabetes Care 199720 1183-1197.

19 Unwin N, Shaw J, Zimmet P \& Alberti KG. Impaired glucose tolerance and impaired fasting glycaemia: the current status on definition and intervention. Diabetic Medicine 200219 708-723. (doi:10.1371/ journal.pone.0065174)

20 Dankner R, Abdul-Ghani MA, Gerber Y, Chetrit A, Wainstein J \& Raz I. Predicting the 20-year diabetes incidence rate. Diabetes/Metabolism Research and Reviews 200723 551-558. (doi:10.1002/(ISSN)1520-7560)

21 Abdul-Ghani MA, Stern MP, Lyssenko V, Tuomi T, Groop L \& Defronzo RA. Minimal contribution of fasting hyperglycemia to the incidence of type 2 diabetes in subjects with normal 2-h plasma glucose. Diabetes Care 201033 557-561. (doi:10.2337/ dc09-1145)

22 Wilson PW, Meigs JB, Sullivan L, Fox CS, Nathan DM \& D Agostino RB Sr. Prediction of incident diabetes mellitus in middle-aged adults: the Framingham Offspring Study. Archives of Internal Medicine 2007167 1068-1074. (doi:10.1089/met.2013.0148)

23 Valleron AJ, Eschwege E, Papoz L \& Rosselin GE. Agreement and discrepancy in the evaluation of normal and diabetic oral glucose tolerance test. Diabetes 197524 585-593. (doi:10.2337/diabetes.24.6.585)

24 American Diabetes A. Standards of medical care in diabetes - 2014. Diabetes Care 2014 37(Supplement 1) S14-S80. (doi:10.2337/ dc14-S014)

25 Abdul-Ghani MA, Abdul-Ghani T, Ali N, Defronzo RA. One-hour plasma glucose concentration and the metabolic syndrome identify subjects at high risk for future type 2 diabetes. Diabetes Care 200831 1650-1655. (doi:10.2337/dc08-0225)

26 Abdul-Ghani MA, Abdul-Ghani T, Stern MP, Karavic J, Tuomi T, Bo I, Defronzo RA \& Groop L. Two-step approach for the prediction of future type 2 diabetes risk. Diabetes Care 201134 2108-2112. (doi:10.2337/dc10-2201)

27 Abdul-Ghani MA, Lyssenko V, Tuomi T, DeFronzo RA \& Groop L. Fasting versus postload plasma glucose concentration and the risk for future type 2 diabetes: results from the Botnia Study. Diabetes Care 200932 281-286. (doi:10.1002/dmrr.v26:4)

28 Abdul-Ghani MA, Williams K, DeFronzo RA \& Stern M. What is the best predictor of future type 2 diabetes? Diabetes Care 200730 1544-1548.

29 Kuang L, Huang Z, Hong Z, Chen A \& Li Y. Predictability of 1-h postload plasma glucose concentration: a 10-year retrospective cohort study. Journal of Diabetes Investigation 20156 647-654. (doi:10.1111/jdi.2015.6.issue-6)

30 Janghorbani $\mathrm{M} \&$ Amini M. Comparison of fasting glucose with post-load glucose values and glycated hemoglobin for prediction of type 2 diabetes: the Isfahan diabetes prevention study. Review of Diabetic Studies 20096 117-123. (doi:10.4103/1735-1995.170596)

31 Gerich JE. Is reduced first-phase insulin release the earliest detectable abnormality in individuals destined to develop type 2 diabetes? Diabetes 200251 (Supplement 1) S117-S121. (doi:10.2337/diabetes.51.2007.S117)

32 Del Prato S, Marchetti P \& Bonadonna RC. Phasic insulin release and metabolic regulation in type 2 diabetes. Diabetes 2002 51(Supplement 1) S109-S116. (doi:10.1097/TP.0b013e318247a782)

33 Karp I, Abrahamowicz M, Bartlett G \& Pilote L. Updated risk factor values and the ability of the multivariable risk score to predict coronary heart disease. American Journal of Epidemiology 2004 160 707-716. (doi:10.1093/aje/kwh258)

34 Saad MF, Knowler WC, Pettitt DJ, Nelson RG, Mott DM \& Bennett PH. The natural history of impaired glucose tolerance in the Pima Indians. 
New England Journal of Medicine 1988319 1500-1506. (doi:10.2337/ diacare.16.4.608)

35 Lorenzo C, Okoloise M, Williams K, Stern MP, Haffner SM \& San Antonio Heart $\mathrm{S}$. The metabolic syndrome as predictor of type 2 diabetes: the San Antonio heart study. Diabetes Care 2003 26 3153-3159. (doi:10.2337/diacare.26.11.3153)

36 Collaboration NCDRF. Effects of diabetes definition on global surveillance of diabetes prevalence and diagnosis: a pooled analysis of 96 population-based studies with 331288 participants. Lancet Diabetes \& Endocrinology 20153 624-637. (doi:10.1016/S2213-8587(15)00129-1)
37 Inzucchi SE. Clinical practice. Diagnosis of diabetes. New England Journal of Medicine 2012367 542-550. (doi:10.1056/ NEJMcp1103643)

38 Balkau B, Soulimane S, Lange C, Gautier A, Tichet J, Vol S \& Desir Studt Group. Are the same clinical risk factors relevant for incident diabetes defined by treatment, fasting plasma glucose, and HbA1c? Diabetes Care 201134 957-959. (doi:10.2337/dc10-1581)

39 Eriksson KF \& Lindgarde F. Prevention of type 2 (non-insulindependent) diabetes mellitus by diet and physical exercise. The 6-year Malmo feasibility study. Diabetologia 199134 891-898.

Received 16 December 2015

Revised version received 8 February 2016

Accepted 11 February 2016 